The hydrodynamic Chaplygin sleigh

This article has been downloaded from IOPscience. Please scroll down to see the full text article.

2010 J. Phys. A: Math. Theor. 43434013

(http://iopscience.iop.org/1751-8121/43/43/434013)

View the table of contents for this issue, or go to the journal homepage for more

Download details:

IP Address: 147.83.132.102

The article was downloaded on 27/11/2012 at 08:19

Please note that terms and conditions apply. 


\title{
The hydrodynamic Chaplygin sleigh
}

\author{
Yuri N Fedorov $^{1}$ and Luis C García-Naranjo ${ }^{2}$ \\ ${ }^{1}$ Department de Matemática Aplicada I, Universitat Politecnica de Catalunya, \\ Barcelona, E-08028, Spain \\ ${ }^{2}$ Section de Mathematiques, Station 8, EPFL, CH-1015 Lausanne, Switzerland \\ E-mail: Yuri.Fedorov@upc.edu and luis.garcianaranjo@epfl.ch
}

Received 3 March 2010, in final form 10 June 2010

Published 12 October 2010

Online at stacks.iop.org/JPhysA/43/434013

\begin{abstract}
We consider the motion of rigid bodies in a potential fluid subject to certain nonholonomic constraints and show that it is described by Euler-PoincaréSuslov equations. In the two-dimensional case, when the constraint is realized by a blade attached to the body, the system provides a hydrodynamic generalization of the classical Chaplygin sleigh problem, one of the best known examples of nonholonomic systems. The dynamics of the generalized sleigh is studied in detail. Namely, the equations of motion are integrated explicitly, and the asymptotic behavior of the system is described analytically and from the qualitative point of view. It is shown that the presence of the fluid brings new features to such a behavior.
\end{abstract}

PACS numbers: $\quad$ 22.20.Sv, 02.30.Ik, 45.50.Dd, 47.15.Km

(Some figures in this article are in colour only in the electronic version)

\section{Introduction and outline}

This paper considers the motion of rigid bodies in a potential fluid in the presence of certain kinds of linear nonholonomic constraints. This is motivated by studying the dynamics of underwater vehicles with large fins, which, in the first approximation, impose restrictions on the velocity of their central points relative to the fluid.

The free motion of such vehicles in a three-dimensional potential fluid can be described by finite-dimensional Kirchhoff equations, in which the action of the fluid is reflected via the tensor of adjoint masses that depends solely on the body shape.

In the presence of a large fin this tensor becomes almost singular: one of its eigenvalues is very large. One of the possible approaches is to take the infinite limit. Namely, as was shown in [13], see also [1] (section 6.4), as the large eigenvalue tends to infinity, the dynamics of the body with such a fin is described by a vakonomic system with the nonholonomic constraint that prohibits the instantaneous motion in the direction corresponding to the singular eigenvalue. 
However, in general, the vakonomic description of mechanical systems with nonholonomic constraints gives rise to rather unexpected (non-physical) behavior (see again [13]).

In our paper we follow another approach, which is more direct. Namely, we describe the motion of the body with a fin by the Kirchhoff equations with a regular tensor of adjoint masses and impose linear constraint(s) on the velocities, which serve to model the action of the fin. The reaction forces arising from the constraint are included according to the Lagrange-D'Alembert principle, which is widely accepted to be physically meaningful.

Our model for the constraint and the reaction forces can also be derived following the anisotropic friction approach for realizing constraints, as described in [13, 19], see also [5]. In this approach one considers the unconstrained system under the influence of a viscous frictional term that only acts in the direction perpendicular to the fin and is proportional to a parameter, say $\zeta$. The equations of motion are then obtained by letting $\zeta \rightarrow \infty$. The obtained system is in general different from the limit vakonomic system described above.

The idea of modeling the action of the fin on the fluid with a nonholonomic constraint using Lagrange-D'Alembert's principle also appears in [23]. Although it is regarded as an idealized situation that is physically unattainable, it is the first approximation for their analysis. The authors use it to study the motion of an underwater projectile with tail fins moving at high speed. However, due to the high speed of the projectile, cavitation effects appear and the general interaction of the fluid and the body motion is not modeled with Kirchhoff's approach.

We thus believe that the systems we present serve as a first approximation for the motion of an underwater vehicle with a large (or very effective) fin that moves in a fluid in the regime where Kirchhoff's approach is valid.

In the case of a two-dimensional body on a plane and two-dimensional fluid, another motivation for the constraint appears: the body can interact with the plane via a sharp blade. This setting gives a hydrodynamic generalization of the famous nonholonomic Chaplygin sleigh problem considered in detail in [6, 19].

This paper is organized as follows. In section 2, we review the preliminaries that are necessary for writing down the equations of motion for our family of systems. We briefly recall Kirchhoff's equations for a rigid body moving in a potential flow and the Euler-PoincaréSuslov equations for nonholonomic systems on Lie groups with left-invariant Lagrangian and constraints. Toward the end of the section the equations of motion for underwater bodies subject to a Suslov or Chaplygin sleigh-type constraint are given. We then discuss the necessary and sufficient conditions for the existence of an invariant measure in a simple case.

The problem of the motion of the Chaplygin sleigh moving in a potential flow (with no circulation) is treated in detail in section 3. The reduced equations are written down explicitly for a general body shape and their qualitative behavior is determined. It is shown that these equations are Hamiltonian with respect to a given bracket but do not preserve a measure with a smooth density in the generic case.

We then continue to show that in the presence of the fluid, the sleigh generically evolves from one asymptotic circular motion to another in the opposite direction, although the limit circles do not coincide. Their radii are given in terms of the components of the total inertia of the fluid-body system. For the purpose of concreteness, the added inertia tensor is computed explicitly for an elliptical sleigh whose contact point $P$ with the plane is located at the center of the ellipse $O$, and where the knife edge is not aligned with its principal axes. This also allows us to calculate the components of this tensor when $P$ does not coincide with $O$.

Then, in the general case, the asymptotic behavior (radius and course direction along the limit circle) is fully determined by the position of the center of mass. 
In section 4 the reduced equations of motion for the hydrodynamic Chaplygin sleigh are integrated explicitly for a generic sleigh. The angular velocity is integrated to give a closed expression for the angle that determines the orientation of the body. Even though the position of the sleigh, as a function of time, cannot be obtained in a closed form, the distance between the centers of the limit circles is computed explicitly.

Finally, in section 5 we motivate a further study of the hydrodynamic Chaplygin sleigh in the presence of circulation and/or point vortices.

\section{Preliminaries}

\subsection{Rigid body motion in a potential flow}

The motion of a rigid body in a potential fluid in the absence of external forces was first described by Kirchhoff in 1890, who derived the reduced equations for the evolution of the body that do not incorporate the fluid itself. Its presence is instead encoded in an 'added inertia' matrix that depends on the body shape.

Kirchhoff's equations can be understood as the output of a two-stage reduction procedure. In the first stage one gets rid of the fluid variables by virtue of the 'particle relabeling symmetry'. In the second stage one eliminates the body configuration variables by homogeneity and isotropy of space. As such, Kirchhoff's equations are the Lie-Poisson equations on the co-algebra $\operatorname{se}(3)^{*}$ where the Hamiltonian is the total energy of the fluid-body system. See $[12,20]$ for more details on this reduction.

We briefly recall Kirchhoff's equations in order to introduce the notation required for the rest of the paper. For a derivation of these equations obtained by balancing the momentum of the body with the forces and torques exerted by the fluid see Lamb's classic book on hydrodynamics [16], which presents a thorough discussion of the problem and remains to date a key reference in the subject.

We adopt Euler's approach to the study of the rigid body dynamics and consider an orthonormal body frame that is attached to the body. This frame is related to a fixed space frame via an attitude (or rotation) matrix $g(t) \in S O(3)$.

Let $\mathbf{V}(t) \in \mathbb{R}^{3}, \boldsymbol{\omega}(t) \in \mathbb{R}^{3}$ be the linear velocity of the origin of the body and its angular velocity, where both vectors are written with respect to the body frame. We then have

$$
\hat{\boldsymbol{\omega}}(t)=g^{-1}(t) \dot{g}(t), \quad \mathbf{V}(t)=g^{-1}(t) \dot{\mathbf{x}}(t),
$$

where the components of $\mathbf{x}(t) \in \mathbb{R}^{3}$ are the spatial coordinates of the origin of the body frame at time $t$, and

$$
\hat{\boldsymbol{\omega}}:=\left(\begin{array}{ccc}
0 & -\omega_{3} & \omega_{2} \\
\omega_{3} & 0 & -\omega_{1} \\
-\omega_{2} & \omega_{1} & 0
\end{array}\right), \quad \boldsymbol{\omega}=\left(\begin{array}{l}
\omega_{1} \\
\omega_{2} \\
\omega_{3}
\end{array}\right), \quad \mathbf{V}=\left(\begin{array}{l}
v_{1} \\
v_{2} \\
v_{3}
\end{array}\right)
$$

The configuration of the body is completely determined by the pair $(g(t), \mathbf{x}(t))$, an element of the Euclidean group $S E(3)$. In this way $\xi:=(\omega, \mathbf{V})$ is thought of as an element of the Lie algebra $\operatorname{se}(3)$ that is identified with $\mathbb{R}^{6}$ via the bracket

$$
[(\boldsymbol{\omega}, \mathbf{V}),(\boldsymbol{\eta}, \mathbf{U})]=(\boldsymbol{\omega} \times \boldsymbol{\eta}, \boldsymbol{\omega} \times \mathbf{U}-\boldsymbol{\eta} \times \mathbf{V}),
$$

where ' $x$ ' denotes the standard vector product in $\mathbb{R}^{3}$.

The kinetic energy of the body is given by (see, e.g., [17])

$$
T_{\mathcal{B}}=\frac{1}{2} \sum_{i=1}^{3}\left(m v_{i}^{2}+\sum_{j=1}^{3} \mathcal{I}_{\mathcal{B}}^{i j} \omega_{i} \omega_{j}+2 \mathcal{K}_{\mathcal{B}}^{i j} v_{i} \omega_{j}\right)
$$


where $m$ is the total mass of the body and the constants $\mathcal{I}_{\mathcal{B}}^{i j}$ and $\mathcal{K}_{\mathcal{B}}^{i j}, i, j=1,2,3$, depend on its shape and mass distribution. Here the $3 \times 3$ matrix $\mathcal{I}_{\mathcal{B}}^{i j}$ is the usual inertia tensor of the body with respect to the chosen frame. If the origin of the body frame is at the center of mass, then $\mathcal{K}_{\mathcal{B}}^{i j}=0$. For convenience we introduce the $6 \times 6$ symmetric matrix

$$
\mathbb{I}_{\mathcal{B}}:=\left(\begin{array}{ll}
\mathcal{I}_{\mathcal{B}} & \mathcal{K}_{\mathcal{B}} \\
\mathcal{K}_{\mathcal{B}}^{T} & m I
\end{array}\right)
$$

( $I$ denoting the $3 \times 3$ identity matrix) that defines $T_{\mathcal{B}}$ as a quadratic form on $s e(3)$.

We now consider the motion of the potential flow that surrounds the body. Suppose that at a given instant the body occupies the compact region $\mathcal{O} \subset \mathbb{R}^{3}$. The fluid motion takes place in the open boundless region $\mathcal{U}=\mathbb{R}^{3} \backslash \mathcal{O}$ that is not occupied by the body. We assume that $\mathcal{U}$ is connected, and that we can write the Eulerian fluid velocity $\mathbf{u}=\nabla \Phi$, for a potential $\Phi: \mathcal{U} \rightarrow \mathbb{R}$. It is also assumed that the fluid is incompressible which implies that $\Phi$ is harmonic on $\mathcal{U}$ :

$$
\nabla^{2} \Phi=0 \quad \text { on } \mathcal{U}
$$

The boundary conditions for $\Phi$ come from the following considerations. On the one hand, it is assumed that the motion of the fluid is solely due to the motion of the body so we require that $\nabla \Phi$ vanishes at infinity. On the other hand, to avoid cavitation or penetration of the fluid into the body, we require the normal component of the fluid velocity at a material point $p$ on the boundary of $\mathcal{O}$ to agree with the normal component of the velocity of $p$. Suppose that the vector $\mathbf{X} \in \mathbb{R}^{3}$ gives body coordinates for $p$. The latter boundary condition is expressed as

$$
\left.\frac{\partial \Phi}{\partial n}\right|_{p \in \partial \mathcal{O}}=(\mathbf{V}+\boldsymbol{\omega} \times \mathbf{X}) \cdot \mathbf{n}
$$

where ' $'$ is the standard Euclidean scalar product and $\mathbf{n}$ denotes the outward unit normal vector to $\mathcal{O}$ at $p$ written in body coordinates.

The potential $\Phi$ satisfying the above boundary value problem can be written in terms of the body's linear and angular velocities V, $\boldsymbol{\omega}$ in the Kirchhoff form:

$$
\Phi=\sum_{i=1}^{3}\left(v_{i} \psi_{i}+\omega_{i} \chi_{i}\right)
$$

where $\psi_{i}$ and $\chi_{i}$ are the harmonic functions on $\mathcal{U}$ whose gradients vanish at infinity and satisfy

$$
\left.\frac{\partial \psi_{i}}{\partial n}\right|_{p \in \partial \mathcal{O}}=\mathbf{n}_{i},\left.\quad \frac{\partial \chi_{i}}{\partial n}\right|_{p \in \partial \mathcal{O}}=(\mathbf{X} \times \mathbf{n})_{i}, \quad i=1,2,3 .
$$

Note that the functions $\psi_{i}, \chi_{i}$ only depend on the shape of the body.

Next, the total energy of the fluid is given by

$$
T_{\mathcal{F}}=\frac{\rho}{2} \int_{\mathcal{U}}\|\mathbf{u}\|^{2} \mathrm{~d} V
$$

where $\rho$ is the (constant) fluid density and $\mathrm{d} V$ is the standard Euclidean volume element in $\mathbb{R}^{3}$. [16])

Putting $\mathbf{u}=\nabla \Phi$ and using (2.3) it is possible to express $T_{\mathcal{F}}$ as the quadratic form (see

$$
T_{\mathcal{F}}=\frac{1}{2} \sum_{i, j=1}^{3}\left(\mathcal{M}_{\mathcal{F}}^{i j} v_{i} v_{j}+\mathcal{I}_{\mathcal{F}}^{i j} \omega_{i} \omega_{j}+2 \mathcal{K}_{\mathcal{F}}^{i j} v_{i} \omega_{j}\right)
$$


where $\mathcal{M}_{\mathcal{F}}^{i j}, \mathcal{I}_{\mathcal{F}}^{i j}$ and $\mathcal{K}_{\mathcal{F}}^{i j}, i, j=1,2,3$, are certain constants that only depend on the body shape (they can be expressed in terms of integrals involving the functions $\psi_{i}$ and $\chi_{i}$ over $\partial \mathcal{O}$ ). They are referred to as added masses and are conveniently written in $6 \times 6$ matrix form to define the added inertia tensor:

$$
\mathbb{I}_{\mathcal{F}}:=\left(\begin{array}{cc}
\mathcal{I}_{\mathcal{F}} & \mathcal{K}_{\mathcal{F}} \\
\mathcal{K}_{\mathcal{F}}^{T} & \mathcal{M}_{\mathcal{F}}
\end{array}\right),
$$

where $\mathcal{I}_{\mathcal{F}}, \mathcal{K}_{\mathcal{F}}$ and $\mathcal{M}_{\mathcal{F}}$ are the corresponding $3 \times 3$ matrices. One can show that the matrix $\mathbb{I}_{\mathcal{F}}$ is symmetric.

In the absence of potential forces, the total energy of the fluid-body system is $T=T_{\mathcal{B}}+T_{\mathcal{F}}$ and defines a kinetic energy Lagrangian $\mathcal{L}: T(S E(3)) \rightarrow \mathbb{R}$. The motion of the body in space is determined by the geodesic motion with respect to the Riemannian metric defined by $\mathcal{L}$.

In view of (2.2) and (2.4), we can write the Lagrangian $\mathcal{L}=T_{\mathcal{B}}+T_{\mathcal{F}}$ in terms of the linear and angular velocities of the body (written in the body frame) and this expression does not depend on the particular position and orientation of the body, i.e. is independent of $(g, \mathbf{x})$. Thus, $\mathcal{L}$ is invariant under the lifted action of left multiplication on $S E(3)$. This symmetry corresponds to invariance under translations and rotations of the space frame. The reduction of this symmetry defines Euler-Poincaré equations on the Lie algebra se(3) or, in the Hamiltonian setting, Lie-Poisson equations on the co-algebra se (3)*. The latter are precisely Kirchhoff's equations that are explicitly written below.

Define the reduced Lagrangian $L: \operatorname{se}(3) \rightarrow \mathbb{R}$ by

$$
L(\xi)=\frac{1}{2} \xi^{T} \mathbb{I} \xi,
$$

where $\xi=(\omega, \mathbf{V}) \in \mathbb{R}^{3} \times \mathbb{R}^{3}$ is thought of as a column vector and the matrix $\mathbb{I}=\mathbb{I}_{\mathcal{B}}+\mathbb{I}_{\mathcal{F}}$. An element $\mu$ in the co-algebra $s e(3)^{*}$ will be represented as a pair $\mu=(\mathbf{k}, \mathbf{p}) \in \mathbb{R}^{3} \times \mathbb{R}^{3}$ and will also be thought of as a six-dimensional column vector. Its action on $\xi=(\boldsymbol{\omega}, \mathbf{V})$ is defined by

$$
\langle\mu, \xi\rangle=\mathbf{k} \cdot \boldsymbol{\omega}+\mathbf{p} \cdot \mathbf{V}
$$

With this identification, the Legendre transform defines the mapping between $s e(3)$ and $s e(3) *$ given by $\mu=\mathbb{I} \xi$. We explicitly have $\mu=(\mathbf{k}, \mathbf{p})$ where

$\mathbf{k}=\left(\mathcal{I}_{\mathcal{B}}+\mathcal{I}_{\mathcal{F}}\right) \boldsymbol{\omega}+\left(\mathcal{K}_{\mathcal{B}}+\mathcal{K}_{\mathcal{F}}\right) \mathbf{V}, \quad \mathbf{p}=m \mathbf{V}+\mathcal{M}_{\mathcal{F}} \mathbf{V}+\left(\mathcal{K}_{\mathcal{B}}^{T}+\mathcal{K}_{\mathcal{F}}^{T}\right) \boldsymbol{\omega}$

In classical hydrodynamics $\mathbf{k}$ and $\mathbf{p}$ are known as 'impulsive pair' and 'impulsive force' respectively.

The reduced Hamiltonian $H: \operatorname{se}(3)^{*} \rightarrow \mathbb{R}$ is given by $H(\mu)=\frac{1}{2} \mu^{T} \mathbb{I}^{-1} \mu$, and the corresponding Lie-Poisson equations $\dot{\mu}=\operatorname{ad}_{\mathbb{I}^{-1} \mu}^{*} \mu$ are then $(\dot{\mathbf{k}}, \dot{\mathbf{p}})=\operatorname{ad}_{(\boldsymbol{\omega}, \mathbf{V})}^{*}(\mathbf{k}, \mathbf{p})$. This gives the Kirchhoff equations

$$
\dot{\mathbf{k}}=\mathbf{k} \times \boldsymbol{\omega}+\mathbf{p} \times \mathbf{V}, \quad \dot{\mathbf{p}}=\mathbf{p} \times \boldsymbol{\omega} .
$$

In the absence of the fluid ( $\rho=0$ ) we have $\mathbb{I}_{\mathcal{F}}=0$. Then, choosing the origin of the body axes at the center of mass, we obtain $\mathcal{K}_{\mathcal{B}}=0$ and $\mathbf{k}=\mathcal{I}_{\mathcal{B}} \boldsymbol{\omega}, \mathbf{p}=m \mathbf{V}$. As a consequence, equations (2.7) decouple, and we recover the well-known fact about the motion of the body in vacuum: the center of mass moves at constant velocity, whereas the body rotates freely according to the Euler equations. It is also well known that in the presence of the fluid this is no longer true, that is, the fluid couples the translational and rotational modes of the motion.

Given a solution of (2.7), the motion of the body in space is obtained by solving the reconstruction equations (2.1).

The description of the problem in the two-dimensional case has a complication with respect to the three-dimensional one. This time the potential flow around the body, satisfying 
the appropriate boundary conditions, is determined up to a purely circulatory flow around the body. To determine the flow uniquely one needs to specify the value of the circulation.

For simplicity, in our paper we will only consider two-dimensional flows with zero circulation, when the motion of the body is described in a manner completely analogous to the three-dimensional case. The configuration space for the body motion is $S E(2)$ and we ultimately get Lie-Poisson equations on $s e(2)^{*}$. This time we write $\xi \in s e(2)$ as $\xi=(\omega, \mathbf{V}) \in \mathbb{R} \times \mathbb{R}^{2}$, and $\mu \in \operatorname{se}(2)^{*}$ as $\mu=(k, \mathbf{p}) \in \mathbb{R} \times \mathbb{R}^{2}$. The pairing between $\mu$ and $\xi$ is the analog of (2.5). Then all the above discussion for the three-dimensional case remains true by simply inserting the appropriate definition of the matrices $\mathbb{I}_{\mathcal{B}}$ and $\mathbb{I}_{\mathcal{F}}$ that relate the column vectors $(k, \mathbf{p})$ and $(\omega, \mathbf{V})$. These are $3 \times 3$ matrices given by

$$
\mathbb{I}_{\mathcal{B}}=\left(\begin{array}{ccc}
\mathcal{I}+m\left(a^{2}+b^{2}\right) & -m b & m a \\
-m b & m & 0 \\
m a & 0 & m
\end{array}\right), \quad \mathbb{I}_{\mathcal{F}}=\left(\begin{array}{cc}
\mathcal{I}_{\mathcal{F}} & \mathcal{K}_{\mathcal{F}} \\
\mathcal{K}_{\mathcal{F}}^{T} & \mathcal{M}_{\mathcal{F}}
\end{array}\right),
$$

where $m$ is the mass of the body, $(a, b)$ are the body coordinates of the center of mass and $\mathcal{I}$ is the moment of inertia of the body about the center of mass. This time $\mathcal{I}_{\mathcal{F}}$ is a scalar, $\mathcal{K}_{\mathcal{F}}$ is a two-dimensional row vector and $\mathcal{M}_{\mathcal{F}}$ is a $2 \times 2$ matrix. As before, the elements of $\mathbb{I}_{\mathcal{F}}$ depend solely on the body shape.

The Lie-Poisson equations $\dot{\mu}=\mathrm{ad}_{\mathbb{I}^{-1} \mu}^{*} \mu$ written in components are

$$
\dot{k}=v_{2} p_{1}-v_{1} p_{2}, \quad \dot{p}_{1}=\omega p_{2}, \quad \dot{p}_{2}=-\omega p_{1},
$$

where $\mathbf{p}=\left(p_{1}, p_{2}\right)$ and $(k, \mathbf{p})=\mathbb{I}(\omega, \mathbf{V})$ with $\mathbb{I}=\mathbb{I}_{\mathcal{B}}+\mathbb{I}_{\mathcal{F}}$. The reconstruction equations (2.1) take the form

$$
\dot{\phi}=\omega, \quad v_{1}=\dot{x} \cos \phi+\dot{y} \sin \phi, \quad v_{2}=-\dot{x} \sin \phi+\dot{y} \cos \phi,
$$

where $\phi$ is the rotation angle between the space and the body frame, and $(x, y)$ are the spatial coordinates of the origin of the body axes.

\subsection{The Euler-Poincaré-Suslov equations}

We have seen that Kirchhoff equations for a rigid body in a potential fluid are Lie-Poisson equations on $s e(3)^{*}\left(s e(2)^{*}\right.$ in the two-dimensional case) corresponding to a pure kinetic energy left-invariant Lagrangian. We are interested in adding left-invariant nonholonomic constraints to the system. The resulting reduced equations that are consistent with Lagrange-D'Alembert's principle, which states that the constraint force performs no work during the motion, are the so-called Euler-Poincaré-Suslov (EPS) equations. We will write these equations explicitly.

In general, a nonholonomic system on a Lie group $G$ with a left-invariant kinetic energy Lagrangian and left-invariant constraints is termed an LL system. Due to invariance, the dynamics reduce to the Lie algebra $\mathfrak{g}$, or to its dual $\mathfrak{g}^{*}$ if working with the momentum formulation.

The reduced Lagrangian $L: \mathfrak{g} \rightarrow \mathbb{R}$ defines the inertia operator $\mathbb{I}: \mathfrak{g} \rightarrow \mathfrak{g}^{*}$ by the relation

$$
L(\xi)=\frac{1}{2}\langle\mathbb{I} \xi, \xi\rangle, \quad \text { for } \quad \xi \in \mathfrak{g},
$$

where $\langle\cdot, \cdot\rangle$ denotes the duality pairing. The reduced Hamiltonian, $H: \mathfrak{g}^{*} \rightarrow \mathbb{R}$, is then given by

$$
H(\mu)=\frac{1}{2}\left\langle\mu, \mathbb{I}^{-1} \mu\right\rangle, \quad \text { for } \quad \mu \in \mathfrak{g}^{*} .
$$

The constraints can be expressed as the annihilator of independent fixed co-vectors $v_{i} \in \mathfrak{g}^{*}$. We say that an instantaneous velocity $\xi \in \mathfrak{g}$ satisfies the constraints if

$$
\left\langle v_{i}, \xi\right\rangle=0, \quad i=1, \ldots, n,
$$


where $n$ is the number of constraints. The constraints are nonholonomic if the set of vectors $\xi \in \mathfrak{g}$ satisfying the above condition do not span a subalgebra of $\mathfrak{g}$.

The reduced EPS equations on $\mathfrak{g}^{*}$ are given by, see e.g. [4],

$$
\dot{\mu}=\mathrm{ad}_{\mathbb{I}^{-1} \mu}^{*} \mu+\sum_{i=1}^{n} \lambda_{i} v_{i},
$$

where the multipliers $\lambda_{i}$ are certain scalars that are uniquely determined by the condition that constraints (2.10) are satisfied.

\subsection{Underwater rigid body with a left-invariant nonholonomic constraint}

We will be interested in the case $G=S E(3)$ with the co-algebra $s e(3)^{*}=(\mathbf{k}, \mathbf{p})$ and $n=1$, which corresponds to the motion of an underwater rigid body subject to a linear, left-invariant and nonholonomic constraint

$$
\mathbf{a} \cdot \boldsymbol{\omega}+\mathbf{F} \cdot \mathbf{V}=0,
$$

a, $\mathbf{F}$ being some constant vectors in the body frame. The constraint is nonholonomic provided that the set of vectors $(\omega, \mathbf{V})$ that satisfy the above condition do not form a subalgebra of se(3).

Then, in view of (2.7), the EPS equations (2.11) become

$$
\begin{aligned}
& \dot{\mathbf{k}}=\mathbf{k} \times \boldsymbol{\omega}+\mathbf{p} \times \mathbf{V}+\lambda \mathbf{a}, \\
& \dot{\mathbf{p}}=\mathbf{p} \times \boldsymbol{\omega}+\lambda \mathbf{F},
\end{aligned}
$$

where $(\mathbf{k}, \mathbf{p})=\mathbb{I}(\omega, \mathbf{V})$ as described by (2.6), the total inertia operator is $\mathbb{I}=\mathbb{I}_{\mathcal{B}}+\mathbb{I}_{\mathcal{F}}$ and the multiplier $\lambda$ is uniquely determined by constraint (2.12). The system thus describes dynamics on the linear subspace $\mathfrak{d} \subset s e(3)$ defined by (2.12) or on its image in $s e(3)^{*}$.

When both vectors $\mathbf{a}, \mathbf{F}$ are non-zero, it is difficult to present a mechanical interpretation of the constraint.

So, we consider two special cases.

(1) $\mathbf{F}=\mathbf{0}$. That is, the constraint is only on the angular velocity: $\mathbf{a} \cdot \boldsymbol{\omega}=0$. Then equations (2.13) represent a hydrodynamic generalization of the classical Suslov problem, see e.g. [24]. The latter describes the motion of a rigid body about a fixed point in the presence of this constraint.

If the origin of the body is at its mass center $\left(\mathcal{K}_{\mathcal{B}}=0\right)$ and in the added masses the translational and rotational components are decoupled $\left(\mathcal{K}_{\mathcal{F}}=0\right)$, then according to (2.6),

$$
\mathbf{k}=\left(\mathcal{I}_{\mathcal{B}}+\mathcal{I}_{\mathcal{F}}\right) \boldsymbol{\omega}, \quad \mathbf{p}=m \mathbf{V}+\mathcal{M}_{\mathcal{F}} \mathbf{V},
$$

and the system (2.13) takes the closed form

$$
\begin{aligned}
& \dot{\mathbf{k}}=\mathbf{k} \times\left(\mathcal{I}_{\mathcal{B}}+\mathcal{I}_{\mathcal{F}}\right)^{-1} \mathbf{k}+\mathbf{p} \times\left(m I+\mathcal{M}_{\mathcal{F}}\right)^{-1} \mathbf{p}+\lambda \mathbf{a}, \\
& \dot{\mathbf{p}}=\mathbf{p} \times\left(\mathcal{I}_{\mathcal{B}}+\mathcal{I}_{\mathcal{F}}\right)^{-1} \mathbf{k} .
\end{aligned}
$$

If we interpret the momentum $\mathbf{p}$ as a vector fixed in space, the above system has the same form as the equations of the Suslov problem in the quadratic potential (ClebschTisserand) field $U=\frac{1}{2} \mathbf{p} \cdot A \mathbf{p}, A=\left(m I+\mathcal{M}_{\mathcal{F}}\right)^{-1}$, which was studied in detail in $[14,25]$ under the assumption that $\mathbf{a}$ is an eigenvector of $\left(\mathcal{I}_{\mathcal{B}}+\mathcal{I}_{\mathcal{F}}\right)^{-1}$, see also [11]. The latter condition is important to guarantee the existence of an invariant measure (see the discussion below).

Apparently, for the general tensor $\mathbb{I}_{\mathcal{B}}+\mathbb{I}_{\mathcal{F}}$ the system (2.13) with the constraint $\mathbf{a} \cdot \boldsymbol{\omega}=0$ has not been studied before. 
(2) $\mathbf{a}=\mathbf{0}$. That is, the constraint is only on the linear velocity of the body, and the system can be interpreted as an underwater version of the three-dimensional Chaplygin sleigh, a rigid body moving in $\mathbb{R}^{3}$ under the condition $\mathbf{F} \cdot \mathbf{V}=0$. This mechanical setting is regarded as an approximate model of an underwater vehicle with a big fin, as was mentioned in the introduction.

Existence of an invariant measure. It is natural to ask whether equations (2.13) and (2.12) possess a smooth invariant measure. This problem has been considered for general EPS equations on Lie algebras of compact groups by Kozlov [15], by Jovanović [10] in the non-compact case and by Zenkov and Bloch [28] for systems with nontrivial shape space. Following [10], the necessary and sufficient condition for equations (2.11) and (2.10) to have such a measure in the case $n=1$ is that the constraint covector $v=v_{1} \in \mathfrak{g}^{*}$ satisfies

$$
\frac{1}{\left\langle\nu, \mathbb{I}^{-1} v\right\rangle} \mathrm{ad}_{\mathbb{I}^{-1} v}^{*} v+T=c v, \quad c \in \mathbb{R},
$$

where $T \in \mathfrak{g}^{*}$ is defined by the relation $\langle T, \xi\rangle=\operatorname{trace}\left(\operatorname{ad}_{\xi}\right), \xi \in \mathfrak{g}$.

Since the group $S E(3)$ is unimodular, in our case $T=0$, and in the case of the generic constraint (2.12) the above condition becomes

$$
(\mathbf{a} \times \mathbf{U}+\mathbf{F} \times \mathbf{W}, \mathbf{F} \times \mathbf{U})=c(\mathbf{a}, \mathbf{F}), \quad c \in \mathbb{R},
$$

where $(\mathbf{U}, \mathbf{W}):=\mathbb{I}^{-1}(\mathbf{a}, \mathbf{F})$.

This condition is easily analyzed in the special cases that we considered (either $\mathbf{F}=\mathbf{0}$ or $\mathbf{a}=\mathbf{0})$ under the assumption that both $\mathcal{K}_{\mathcal{B}}=0$ and $\mathcal{K}_{\mathcal{F}}=0$. One can then show that (2.14) is equivalent to asking that $\mathbf{a}$ is an eigenvector of $\left(\mathcal{I}_{\mathcal{B}}+\mathcal{I}_{\mathcal{F}}\right)^{-1}$ in the case $\mathbf{F}=\mathbf{0}$, or to the condition that $\mathbf{F}$ is an eigenvector of $A=\left(m I+\mathcal{M}_{\mathcal{F}}\right)^{-1}$ in the case $\mathbf{a}=\mathbf{0}$.

\section{The hydrodynamic planar Chaplygin sleigh}

We now consider in detail the two-dimensional version of (2.13), which corresponds to the co-algebra se $(2)^{*}=(k, \mathbf{p})$ and the nonholonomic constraint

$$
a \omega+F_{1} v_{1}+F_{2} v_{2}=0
$$

Assume that $a=0$ and choose, without loss of generality, $F_{1}=0$. Then the constraint takes the form $v_{2}=0$ and the reduced equations of motion are

$$
\dot{k}=v_{2} p_{1}-v_{1} p_{2}, \quad \dot{p}_{1}=\omega p_{2}, \quad \dot{p}_{2}=-\omega p_{1}+\lambda .
$$

Here the column vectors $(k, \mathbf{p})^{T}$ and $(\omega, \mathbf{V})^{T}$ are related by $(k, \mathbf{p})^{T}=\mathbb{I}(\omega, \mathbf{V})^{T}$, with the $3 \times 3$ tensor $\mathbb{I}=\mathbb{I}_{\mathcal{B}}+\mathbb{I}_{\mathcal{F}}$. The multiplier $\lambda$ is determined by the condition $v_{2}=0$.

In the absence of fluid $\left(\mathbb{I}_{\mathcal{F}}=0\right.$ ) equations (3.1) become the classical Chaplygin sleigh problem, which goes back to 1911 [6] and describes the motion of a planar rigid body with a knife edge (a blade) that slides on the plane. The nonholonomic constraint forbids the motion in the direction perpendicular to the knife edge. The asymptotic motions of the sleigh on the plane are straight-line uniform motions, see $[4,6,19]$.

In the presence of the potential fluid $\left(\mathbb{I}_{\mathcal{F}} \neq 0\right)$ the added masses define a more general kinetic energy, and the system (3.1) describes a hydrodynamic generalization of the Chaplygin sleigh. We shall see that this leads to new features in the asymptotic behavior of the body.

The total inertia tensor. Introduce the body reference frame $\left\{\mathbf{E}_{\mathbf{1}} \mathbf{E}_{\mathbf{2}}\right\}$ centered at the contact point between the knife edge and the plane and choose $\mathbf{E}_{\mathbf{1}}$ to be parallel to the blade and $\mathbf{E}_{\mathbf{2}}$ orthogonal to it. This ensures the above constraint $v_{2}=0$. 


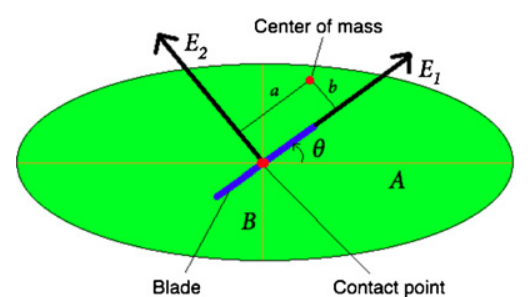

(a)

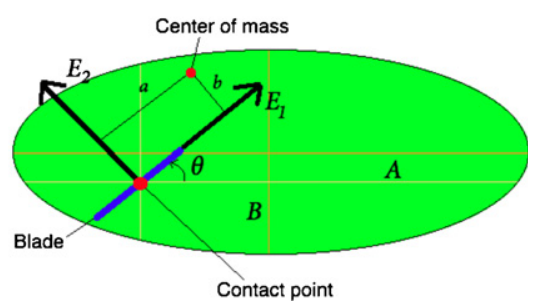

(b)

Figure 1. The elliptical sleigh. The blade makes an angle $\theta$ with the major axis of the ellipse.

While the expression for $\mathbb{I}_{\mathcal{B}}$ with respect to the body frame was given in (2.8) for an arbitrary body, the expression for the tensor of adjoint masses $\mathbb{I}_{\mathcal{F}}$ can be given explicitly only for rather simple geometries. A simple yet interesting case is an elliptical planar body with semi-axes $A>B>0$. First assume that the origin is at the center of the ellipse, but the coordinate axes $\mathbf{E}_{\mathbf{1}}, \mathbf{E}_{\mathbf{2}}$ are not aligned with the axes of the ellipse, forming an angle $\theta$ (measured counter-clockwise), as illustrated in figure 1(a).

For this geometry, using the formula for the fluid potential $\Phi$ given in [16], one can show that

$$
\mathbb{I}_{\mathcal{F}}=\rho \pi\left(\begin{array}{ccc}
\frac{\left(A^{2}-B^{2}\right)^{2}}{4} & 0 & 0 \\
0 & B^{2} \cos ^{2} \theta+A^{2} \sin ^{2} \theta & \frac{A^{2}-B^{2}}{2} \sin (2 \theta) \\
0 & \frac{A^{2}-B^{2}}{2} \sin (2 \theta) & A^{2} \cos ^{2} \theta+B^{2} \sin ^{2} \theta
\end{array}\right)
$$

The total inertia tensor, $\mathbb{I}=\mathbb{I}_{\mathcal{B}}+\mathbb{I}_{\mathcal{F}}$, of the fluid-body system is then given by

$$
\mathbb{I}=\left(\begin{array}{ccc}
\mathcal{I}+m\left(a^{2}+b^{2}\right)+\rho \pi \frac{\left(A^{2}-B^{2}\right)^{2}}{4} & -m b & m a \\
-m b & m+\rho \pi\left(B^{2} \cos ^{2} \theta+A^{2} \sin ^{2} \theta\right) & \rho \pi\left(\frac{A^{2}-B^{2}}{2}\right) \sin (2 \theta) \\
m a & \rho \pi\left(\frac{A^{2}-B^{2}}{2}\right) \sin (2 \theta) & m+\rho \pi\left(A^{2} \cos ^{2} \theta+B^{2} \sin ^{2} \theta\right)
\end{array}\right) .
$$

Note that in the presence of the fluid, if $\theta \neq n \frac{\pi}{2}, n \in \mathbb{Z}$, the coefficient $\mathbb{I}_{23}=\mathbb{I}_{32}$ is non-zero. This can never be the case if the sleigh is moving in vacuum as one can see from the expression given for $\mathbb{I}_{\mathcal{B}}$ in (2.8). The appearance of this non-zero term leads to interesting dynamics that are studied below and that, to our knowledge, had not been described before in the literature.

Now, if the origin of $\left(\mathbf{E}_{\mathbf{1}} \mathbf{E}_{\mathbf{2}}\right)$ is not in the center of the ellipse (figure 1(b)), then the tensor (3.2) takes a more general form with $\left(\mathbb{I}_{\mathcal{F}}\right)_{13},\left(\mathbb{I}_{\mathcal{F}}\right)_{23}$ non-zero, which can be calculated explicitly and lead to the corresponding modification of the total tensor $\mathbb{I}$.

In the following we assume that the shape of the sleigh is arbitrary convex and that its center of the mass does not necessarily coincide with the origin, which leads to the general total inertia tensor

$$
\mathbb{I}=\left(\begin{array}{ccc}
J & -L_{2} & L_{1} \\
-L_{2} & M & Z \\
L_{1} & Z & N
\end{array}\right) .
$$

We keep in mind that we expect to see new phenomena due to the presence of the fluid (when $Z \neq 0$ ). The tensor for classical Chaplygin sleigh is recovered by setting $Z=0$, $J=\mathcal{I}+m\left(a^{2}+b^{2}\right), M=N=m, L_{1}=m a$ and $L_{2}=m b$. 
Note that, in any case, since the total energy of the motion is positive definite, the tensor II has the same property.

Detailed equations of motion. The constraint written in terms of momenta is $v_{2}=$ $\left(\mathbb{I}^{-1}(k, \mathbf{p})^{T}\right)_{3}=0$. Differentiating it and using (3.1), we find the multiplier

$$
\lambda=-\frac{1}{\left(\mathbb{I}^{-1}\right)_{33}}\left(\mathbb{I}^{-1}\left(\begin{array}{c}
v_{2} p_{1}-v_{1} p_{2} \\
\omega p_{2} \\
-\omega p_{1}
\end{array}\right)\right)_{3}
$$

where

$$
\mathbb{I}^{-1}=\frac{1}{\operatorname{det}(\mathbb{I})}\left(\begin{array}{ccc}
M N-Z^{2} & Z L_{1}+N L_{2} & -Z L_{2}-M L_{1} \\
Z L_{1}+N L_{2} & J N-L_{1}^{2} & -L_{1} L_{2}-J Z \\
-Z L_{2}-M L_{1} & -L_{1} L_{2}-J Z & J M-L_{2}^{2}
\end{array}\right)
$$

A long but straightforward calculation shows that by expressing $\omega, v_{1}$ and $v_{2}$ in terms of $k, p_{1}, p_{2}$, substituting into (3.1) and enforcing the constraint $v_{2}=0$, one obtains

$$
\begin{aligned}
& \dot{\omega}=\frac{1}{D}\left(L_{1} \omega+Z v_{1}\right)\left(L_{2} \omega-M v_{1}\right), \\
& \dot{v}_{1}=\frac{1}{D}\left(L_{1} \omega+Z v_{1}\right)\left(J \omega-L_{2} v_{1}\right),
\end{aligned}
$$

where we set $D=\operatorname{det}(\mathbb{I})\left(\mathbb{I}^{-1}\right)_{33}=M J-L_{2}^{2}$. Note that $D>0$ since $\mathbb{I}$ and $\mathbb{I}^{-1}$ are positive definite.

The full motion of the sleigh on the plane is determined by the reconstruction equations (2.9), which, in our case with $v_{2}=0$, reduce to

$$
\dot{\phi}=\omega, \quad \dot{x}=v_{1} \cos \phi, \quad \dot{y}=v_{1} \sin \phi .
$$

The reduced energy integral has

$$
H=\frac{1}{2}\left(J \omega^{2}+M v_{1}^{2}-2 L_{2} \omega v_{1}\right),
$$

and its level sets are ellipses on the $\left(\omega v_{1}\right)$ plane. As seen from the equations, the straight line $\ell=\left\{L_{1} \omega+Z v_{1}=0\right\}$ consists of equilibrium points for the system.

Hence, if $L_{1}$ and $Z$ do not vanish simultaneously, the trajectories of (3.3) are elliptic arcs that form heteroclinic connections between the asymptotically unstable and stable equilibria on $\ell$ (see figure 2(a)). The phase portrait is similar to that of the classical Chaplygin sleigh except that the line of equilibra is no longer the $v_{1}$ axis if $Z \neq 0$.

Remark. In fact, the reduced two-dimensional system (3.3) can be checked to be Hamiltonian with respect to the following Poisson bracket of functions of $\omega, v_{1}{ }^{3}$

$$
\left\{F_{1}, F_{2}\right\}:=-\frac{1}{D}\left(L_{1} \omega+Z v_{1}\right)\left(\frac{\partial F_{1}}{\partial \omega} \frac{\partial F_{2}}{\partial v_{1}}-\frac{\partial F_{1}}{\partial v_{1}} \frac{\partial F_{2}}{\partial \omega}\right) .
$$

The invariant symplectic leaves consist of the semi-planes separated by the equilibria line $\ell$ and the zero-dimensional leaves formed by the points on this line. The above bracket can be obtained using the construction developed in [8].

Case $Z=L_{1}=0$. We start by considering the most degenerate case when both $L_{1}$ and $Z$ vanish. In particular, for the elliptical sleigh this is the case when $\theta=0$ and $a=0$ in figure 1(a). Then all solutions of (3.3) are equilibria. Such a sleigh always performs a uniform circular or straight-line motion whose parameters depend on the initial condition. In this case, the Euclidean measure (or any smooth measure for that matter) is trivially preserved by 


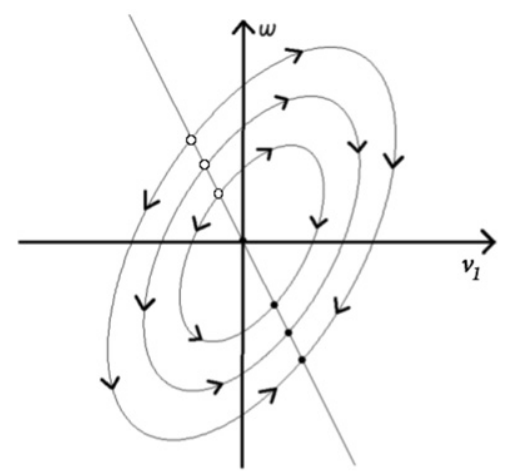

(a)

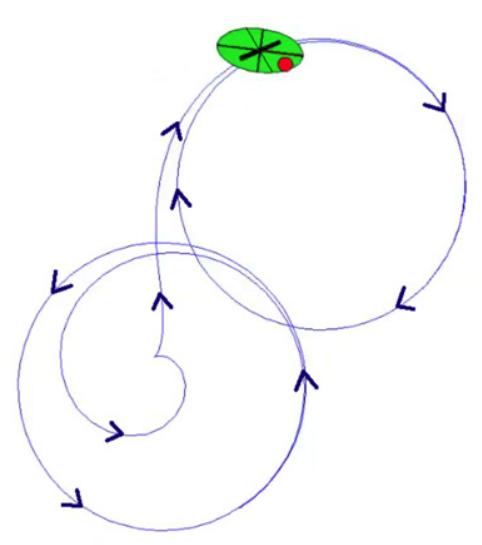

(b)

Figure 2. Phase portrait of the reduced system and trajectory of the sleigh in the plane. (a) Reduced phase portrait under the assumption $L_{1}, Z>0$. The stable and unstable equilibra are represented by filled and empty dots, respectively. $(b)$ Trajectory of the elliptic sleigh on the plane displaying asymptotic evolution from one circular motion to another one in opposite directions. The dot on the sleigh surface represents its center of mass.

equations (3.3). In fact this is the only case in which there is a smooth preserved measure as we now show.

Proposition 3.1. The reduced equations (3.3) possess a smooth invariant measure if and only if $L_{1}=Z=0$.

Proof. The general condition (2.14) for a preserved measure can be specialized to our twodimensional problem by putting

$$
\begin{aligned}
& \mathbf{a}=\mathbf{0}, \quad \mathbf{F}=(0,1,0), \quad \mathbf{U}=\operatorname{det}(\mathbb{I})^{-1}\left(-Z L_{2}-M L_{1}, 0,0\right), \\
& \mathbf{W}=\operatorname{det}(\mathbb{I})^{-1}\left(-L_{1} L_{2}-J Z, J M-L_{2}^{2}, 0\right) .
\end{aligned}
$$

One gets the necessary and sufficient conditions

$$
M L_{1}+Z L_{2}=0, \quad L_{1} L_{2}+J Z=0
$$

The above can be seen as a linear system of equations for $L_{1}$ and $Z$ with non-zero determinant $M J-L_{2}^{2}=D>0$. Hence this condition can only hold if $L_{1}=Z=0$.

The result of this proposition is to be expected from the qualitative behavior of the system that was described above in the case where $L_{1}$ and $Z$ do not vanish simultaneously.

Case $Z \neq 0$. In this case the equilibrium points correspond to the periodic circular motion of the body on the plane, and the contact point of the blade (the origin) goes along circles of the radius

$$
r=\left|\lim _{t \pm \infty} v_{1} / \lim _{t \pm \infty} \omega\right|=\left|-\frac{L_{1}}{Z}\right|,
$$

whereas the whole motion is an asymptotic evolution from one circular motion to the other one, but in opposite directions, as shown in figure $2(b)$. (Clearly, when $L_{1}=0$ the radius $r$ is zero, and the limit motions of the body are just rotations about the fixed origin.)

3 A similar observation for some other generalizations of the Chaplygin sleigh was made in [3]. 
The preferred direction of rotation is determined by the following proposition whose proof follows from a simple linear stability analysis.

Proposition 3.2. Let the line of equilibra $\ell=\left\{L_{1} \omega+Z v_{1}=0\right\}$ be parameterized by $v_{1}(s)=L_{1} s, \omega(s)=-Z s, s \in \mathbb{R}$. The equilibra corresponding to $s<0$ are unstable, whereas the equilibra corresponding to $s>0$ are stable.

Proof. The matrix associated with the linearization about the equilibrium $v_{1}=-L_{1} s, \omega=$ $Z s$, is

$$
-s\left(\begin{array}{cc}
\left(L_{2} Z+M L_{1}\right) L_{1} & \left(L_{2} Z+M L_{1}\right) Z \\
\left(J Z+L_{1} L_{2}\right) L_{1} & \left(J Z+L_{1} L_{2}\right) Z
\end{array}\right) .
$$

It is seen that this matrix has eigenvalues $\lambda_{1}=0$ (corresponding to the continuum of equilibra along the line $\left.\ell=\left\{L_{1} \omega+Z v_{1}=0\right\}\right)$, and $\lambda_{2}=-s E$ with

$$
E=J Z^{2}+2 L_{1} L_{2} Z+M L_{1}^{2}=\left(Z,-L_{1}, 0\right) \mathbb{I}\left(Z,-L_{1}, 0\right)^{T}>0,
$$

since $\mathbb{I}$ is positive definite. Thus $\lambda_{2}$ is positive (negative) if $s<0(s>0)$, corresponding to the unstable (stable) direction.

In particular, due to the above proposition and (3.5), the balanced elliptical sleigh depicted in figure $1(a)$, with $a>0$ and $0<\theta<\frac{\pi}{2}$, will have a limiting motion in the clockwise direction on a circle of radius $r=\frac{2 m a}{\rho \pi\left(A^{2}-B^{2}\right) \sin (2 \theta)}$ as $t \rightarrow \infty$. Note that these conclusions on the qualitative asymptotic behavior of the system are independent of $b$, a feature that is reminiscent of the classical unbalanced Chaplygin sleigh (see [19] and the discussion at the end of section 4). However, we shall see (theorem 4.2) that the distance between the centers of the circles does depend on $b$. The conclusions of the proposition are also illustrated in figure 2.

\section{Explicit solution and asymptotic data}

The general solution of the reduced system (3.3) can be written in the form

$$
\begin{aligned}
& \omega(t)=A\left(\alpha \tanh (A t)+\sigma c_{1} \operatorname{sech}(A t)\right), \\
& v_{1}(t)=A\left(\beta \tanh (A t)+\sigma c_{2} \operatorname{sech}(A t)\right),
\end{aligned}
$$

where the constants

$$
\begin{aligned}
& \alpha=-\frac{D Z}{E}, \quad \beta=\frac{D L_{1}}{E}, \quad c_{1}=\sqrt{D} \frac{Z L_{2}+M L_{1}}{E}, \\
& c_{2}=\sqrt{D} \frac{L_{1} L_{2}+Z J}{E}, \quad E=J Z^{2}+2 Z L_{1} L_{2}+M L_{1}^{2},
\end{aligned}
$$

only depend on the components of the inertia tensor $\mathbb{I}$. Here $\sigma= \pm 1$ corresponding to the two different branches of the trajectories on the phase portrait.

Note that the denominator $E>0$, as shown in (3.6) and that the arbitrary constant $A \geqslant 0$ is related to the energy $H$ of the system by

$$
H=\frac{1}{2}\left(\frac{D^{2}}{E}\right) A^{2} .
$$


The motion on the plane in the general case $Z \neq 0$. In view of (3.4), the angle $\phi$ is calculated by integrating the first expression in (4.1), which yields

$$
\begin{aligned}
& \phi(t)=\phi_{1}+\phi_{2}, \\
& \phi_{1}=\int A \sigma c_{1} \operatorname{sech}(A t) \mathrm{d} t=2 \sigma c_{1}\left(\arctan \left(e^{A t}\right)-\pi / 4\right), \\
& \phi_{2}=\int A \alpha \tanh (A t) \mathrm{d} t=\alpha \ln (\cosh (A t))+\phi_{0},
\end{aligned}
$$

$\phi_{0}$ being an integration constant. The angles $\phi_{1}$ and $\phi_{2}$ are an odd and an even function of $t$, respectively. One can observe that they have quite different behavior:

$$
\lim _{t \rightarrow \pm \infty} \phi_{1}= \pm \sigma \frac{c_{1} \pi}{2}= \pm \sigma \frac{\pi}{2} \frac{\sqrt{D}\left(Z L_{2}+M L_{1}\right)}{E}
$$

whereas, as $t \rightarrow \pm \infty$, the angle $\phi_{2}$ asymptotically approaches the linear function $l(t)=$ $\pm A \alpha t+\phi_{0}-\alpha \ln 2$.

The trajectory of the origin on the plane $(x, y)$ is then described by rather complicated integrals, which themselves do not say much about its properties. However, it is natural to calculate the distance between the centers of the limit circles. To do this, we shall use

Proposition 4.1. The centers of the limit circles coincide with the limit positions of the material point $C$, which in the body reference frame $\left(\mathbf{E}_{\mathbf{1}} \mathbf{E}_{\mathbf{2}}\right)$ has fixed coordinates $(0, \beta / \alpha)=$ $\left(0,-L_{1} / Z\right)$.

Proof. This can be easily obtained from formula (3.5). Alternatively, in view of (4.1), (4.2), the $x$ - and $y$-velocities of the point $C$ are

$$
\begin{aligned}
& \dot{x}_{C}=\left(v_{1}-\frac{\beta}{\alpha} \omega\right) \cos (\phi(t))=A \sigma\left(\frac{\sqrt{D}}{Z}\right) \operatorname{sech}(A t) \cos (\phi(t)), \\
& \dot{y}_{C}=\left(v_{1}-\frac{\beta}{\alpha} \omega\right) \sin (\phi(t))=A \sigma\left(\frac{\sqrt{D}}{Z}\right) \operatorname{sech}(A t) \sin (\phi(t)),
\end{aligned}
$$

and both tend to zero as $t \rightarrow \pm \infty$. Hence $C$ coincides with the centers of the circumferences, since otherwise its limit velocity would not be zero.

Setting $\phi=\phi_{1}+\phi_{2}$ as in (4.3), we obtain the following expressions for the components of the vector $\left(\Delta x_{C}, \Delta y_{C}\right)$ connecting the centers of the limit circumferences:

$$
\begin{aligned}
& \Delta x_{C}=\int_{-\infty}^{\infty} \dot{x}_{C} \mathrm{~d} t=\frac{\sigma \sqrt{D}}{Z} \int_{-\infty}^{\infty} A \operatorname{sech}(A t)\left(\cos \phi_{1} \cos \phi_{2}-\sin \phi_{1} \sin \phi_{2}\right) \mathrm{d} t, \\
& \Delta y_{C}=\int_{-\infty}^{\infty} \dot{y}_{C} \mathrm{~d} t=\frac{\sigma \sqrt{D}}{Z} \int_{-\infty}^{\infty} A \operatorname{sech}(A t)\left(\sin \phi_{1} \cos \phi_{2}+\cos \phi_{1} \sin \phi_{2}\right) \mathrm{d} t
\end{aligned}
$$

Since $\phi_{1}$ is an odd and $\phi_{2}$ is an even function of time, the integrals are reduced to

$$
\begin{aligned}
\Delta x_{C} & =\frac{\sigma \sqrt{D}}{Z} \int_{-\infty}^{\infty} A \operatorname{sech}(A t) \cos \phi_{1} \cos \phi_{2} \mathrm{~d} t \\
\Delta y_{C} & =\frac{\sigma \sqrt{D}}{Z} \int_{-\infty}^{\infty} A \operatorname{sech}(A t) \cos \phi_{1} \sin \phi_{2} \mathrm{~d} t
\end{aligned}
$$


and, if we set $T=A t$,

$\Delta x_{C}=\frac{\sigma \sqrt{D}}{Z} \int_{-\infty}^{\infty} \operatorname{sech}(T) \cos \left(2 c_{1}\left(\arctan \left(e^{T}\right)-\pi / 4\right)\right) \cos \left(\alpha \ln (\cosh T)+\phi_{0}\right) \mathrm{d} T$,

$\Delta y_{C}=\frac{\sigma \sqrt{D}}{Z} \int_{-\infty}^{\infty} \operatorname{sech}(T) \cos \left(2 c_{1}\left(\arctan \left(e^{T}\right)-\pi / 4\right)\right) \sin \left(\alpha \ln (\cosh T)+\phi_{0}\right) \mathrm{d} T$,

$c_{1}, \alpha$ being specified in (4.2).

It follows that, like the radii of the limit circumferences, the distance $d$ between their centers does not depend on the energy, but only on the components of the generalized inertia tensor, as expected ${ }^{4}$.

The length scale of this distance is given by the ratio $\sqrt{D} /|Z|$ and it depends parametrically on the dimensionless quantities $\alpha$ and $c_{1}$. The explicit dependence of the distance $d$ on the parameters $\alpha$ and $c_{1}$ is given by the following theorem whose proof is given in the appendix.

Theorem 4.2. The square of the distance d between the limit circumferences is given by

$$
d^{2}=\left(\Delta x_{C}\right)^{2}+\left(\Delta y_{C}\right)^{2}=\frac{2 \pi D}{Z^{2}}\left(\frac{\alpha}{c_{1}^{2}+\alpha^{2}}\right)\left(\frac{\cosh (\alpha \pi)-\cos \left(c_{1} \pi\right)}{\sinh (\alpha \pi)}\right) .
$$

We mention that formula (4.6) perfectly corresponds to the results of numerical tests.

Case $Z=0, L_{1} \neq 0$. The condition $Z=0$ corresponds to the absence of the fluid, or to the case when the blade is parallel to one of the two specific perpendicular directions in the body frame with the following property: if the solid is set in motion parallel to one of these, without rotation, it will continue to move in this manner. In particular, for the elliptical sleigh, the two directions are precisely the principal axes of the ellipse.

In this case the system reduces to the classical Chaplygin sleigh, whose motion on the plane was described in detail in [19]. Namely, this implies $\alpha=0$ and the line of equilibria on the phase plane $\left(\omega, v_{1}\right)$ is the axis $\omega=0$. The trajectory of the contact point on the plane in this case necessarily has a return point and, in view of (3.4), is given by the rather complicated integrals. However, the limit behaviors of the sleigh are straight-line uniform motions and, according to (4.4), the angle between the limit lines is ${ }^{5}$

$$
\Delta \phi=\sigma c_{1} \pi=\sigma \pi \frac{\sqrt{D}\left(M L_{1}\right)}{E} .
$$

Note that $\Delta \phi$ and $\omega$ have the same sign (the latter does not change throughout the motion).

In the absence of the fluid, this expression becomes

$$
\Delta \phi=\sigma \pi \frac{\sqrt{m\left(\mathcal{I}+m a^{2}\right)}}{m a},
$$

which does not depend on $b$. Figure 3 shows the trajectory of the sleigh in this case for different values of $\Delta \phi$ (see also [19]). Note that $\left[\frac{|\Delta \phi|}{2 \pi}\right]$ is the number of 'loops' that the sleigh performs in its transition between the limit straight-line motions.

\footnotetext{
4 In view of the similarity of the reduced hydrodynamic Chaplygin sleigh and the nonholonomic Suslov problem, the distance $d$ can be regarded as an analog of the angle between the axes of the limit permanent rotations of the Suslov rigid body in space.

5 Here $\Delta \phi$ is measured from the line of asymptotic straight-line motion in the past to the line of the asymptotic straight-line motion in the future in the trigonometric sense.
} 


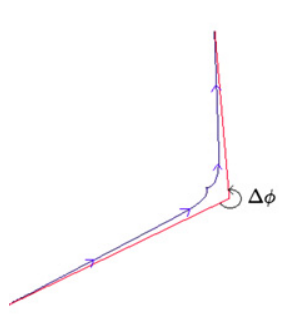

(a) $0<\Delta \phi<2 \pi$

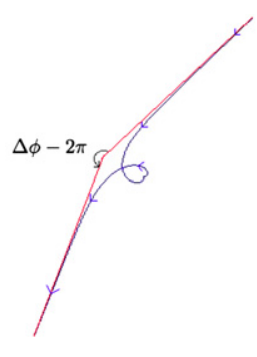

(b) $2 \pi<\Delta \phi<4 \pi$

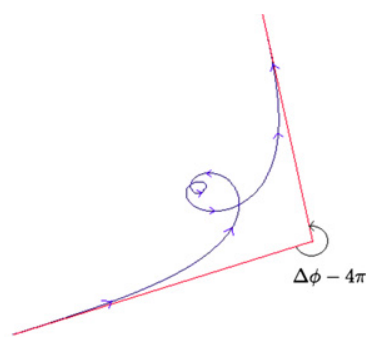

(c) $4 \pi<\Delta \phi<6 \pi$

Figure 3. Trajectory of the contact point of the sleigh on the plane in the case $Z=0$ for different values of $\Delta \phi$. In all these cases $\omega>0$, so $\phi$ is an increasing function of time and the sleigh turns counterclockwise.

\section{Conclusions and further work}

A new series of examples of nonholonomic systems has been presented. These are of interest from the point of view of applications in the design of underwater vehicles and mechanisms, since, as we have mentioned, the constraint can be interpreted as a simple model for a fin.

From the mathematical point of view, our systems provide a motivation to study the problem of nonholonomic geodesics on the group $S E(n)$ with a general left-invariant kinetic energy metric.

The hydrodynamical version of the Chaplygin sleigh that has been considered provides a new example of a simple, integrable nonholonomic system with an interesting asymptotic behavior. Its extensive analysis that we have presented can be of interest in the design of control mechanisms, see [21].

It should be emphasized that we have only considered the case of zero circulation of the fluid. Our preliminary studies show that in the presence of circulation the corresponding equations of motion are no longer of EPS type on the co-algebra $s e(2)^{*}$, but rather on the co-algebra of a central extension of $S E(2)$. However, some of the features of the asymptotic motion remain. In particular, for certain initial conditions, one has asymptotic evolution from one circular motion to another, as before, but the radii of the limit circles are not the same.

In this spirit, another interesting problem to consider is to couple the motion of the nonholonomic sleigh with point vortices. Such a problem (without nonholonomic constraints) has received interest in the past few years, see for example [2, 22, 26].

We hope to report with progress on the problems described above in the near future.

\section{Acknowledgments}

YuF acknowledges the support of MCyT-FEDER grant MTM2006-00478 and grant MTM2009-12670 of the Spanish Ministry of Science and Technology and is grateful to the School of Mathematics of Ecole Politechnique Fédérale de Lausanne for its hospitality during his recent stay there. LGN acknowledges the hospitality at the Department de Matemática Aplicada I, at UPC Barcelona for his recent stay there. The authors are also grateful to Irina Kukk for her help with checking the derivation of the formula of theorem 4.2 and to Maria 
Pzybulska, Joris Vankerschaver, and Dmitry Zenkov for useful discussions and indicating us some relevant references.

\section{Appendix. Proof of theorem 4.2}

Assume without loss of generality that $\phi_{0}=\alpha \ln 2$ in (4.3) and (4.5). This restriction causes a rotation of the vector $\left(\Delta x_{C}, \Delta y_{C}\right)$, but does not affect its length.

First, for simplicity, set $c_{1}=0$, that is, $\left(\mathbb{I}^{-1}\right)_{13}=0$. Then, in view of (4.3), $\phi_{1} \equiv 0$, and the integrals (4.5) give

$$
\begin{aligned}
\Delta x_{C}+\mathrm{i} \Delta y_{C} & =2 \frac{\sigma \sqrt{D}}{Z} \int_{-\infty}^{\infty} \frac{\exp \left(\mathrm{i} \alpha \ln \left(e^{T}+e^{-T}\right)\right)}{e^{T}+e^{-T}} \mathrm{~d} T=\left\{z=e^{T}\right\} \\
& =2 \frac{\sigma \sqrt{D}}{Z} \int_{0}^{\infty} \frac{\exp (\mathrm{i} \alpha \ln (z+1 / z))}{z^{2}+1} \mathrm{~d} z=\{u=\arctan z\} \\
& =2 \frac{\sigma \sqrt{D}}{Z} \int_{0}^{\pi / 2} \exp \left(\mathrm{i} \alpha\left[\ln \left(1+\tan ^{2} u\right)-\ln (\tan u)\right]\right) \mathrm{d} u \\
& =2 \frac{\sigma \sqrt{D}}{Z} \int_{0}^{\pi / 2} \exp \left(\mathrm{i} \alpha \ln \left(\frac{\sec ^{2} u}{\tan u}\right)\right) \mathrm{d} u \\
& =2 \frac{\sigma \sqrt{D}}{Z} \int_{0}^{\pi / 2}(\cos u)^{-\mathrm{i} \alpha}(\sin u)^{-\mathrm{i} \alpha} \mathrm{d} u
\end{aligned}
$$

and, similarly,

$$
\Delta x_{C}-\mathrm{i} \Delta y_{C}=2 \frac{\sigma \sqrt{D}}{Z} \int_{0}^{\pi / 2}(\cos u)^{\mathrm{i} \alpha}(\sin u)^{\mathrm{i} \alpha} \mathrm{d} u
$$

The last integrals have the form of the Euler Beta-function (see e.g., [7])

$$
B(x, y)=\int_{0}^{1} t^{x-1}(1-t)^{y-1} \mathrm{~d} t=2 \int_{0}^{\pi / 2}(\cos u)^{2 x-1}(\sin u)^{2 y-1} \mathrm{~d} u=\frac{\Gamma(x) \Gamma(y)}{\Gamma(x+y)},
$$

$\Gamma(\cdot)$ being the Euler Gamma-function with the properties

$$
\begin{aligned}
& \Gamma\left(\frac{1}{2}-z\right) \Gamma\left(\frac{1}{2}+z\right)=\frac{\pi}{\cos (\pi z)}, \quad \Gamma(-z) \Gamma(z)=-\frac{\pi}{z \sin (\pi z)}, \\
& \Gamma(z+1)=z \Gamma(z), \quad z \in \mathbb{C} .
\end{aligned}
$$

Then (A.1) and (A.2) read

$\Delta x_{C} \pm \mathrm{i} \Delta y_{C}=2 \frac{\sigma \sqrt{D}}{Z} \frac{1}{2} B(1 / 2 \mp \mathrm{i} \alpha / 2,1 / 2 \mp \mathrm{i} \alpha / 2)=\frac{\sigma \sqrt{D}}{Z} \frac{\Gamma^{2}(1 / 2 \mp \mathrm{i} \alpha / 2)}{\Gamma(1 \mp \mathrm{i} \alpha)}$

and, in view of (A.4), the square of the distance is

$$
\begin{aligned}
d^{2} & =\left(\Delta x_{C}\right)^{2}+\left(\Delta y_{C}\right)^{2}=\frac{D}{Z^{2}} \frac{\left[\Gamma\left(\frac{1}{2}-\mathrm{i} \frac{\alpha}{2}\right) \Gamma\left(\frac{1}{2}+\mathrm{i} \frac{\alpha}{2}\right)\right]^{2}}{\Gamma(1+\mathrm{i} \alpha) \Gamma(1-\mathrm{i} \alpha)}=\frac{D}{Z^{2}} \frac{\pi / \cos ^{2}(\pi \mathrm{i} \alpha / 2)}{\mathrm{i} \alpha / \sin (\pi \mathrm{i} \alpha)} \\
& =\frac{D}{Z^{2}} \frac{2 \pi \sin (\pi \mathrm{i} \alpha)}{\mathrm{i} \alpha(1+\cos (\pi \mathrm{i} \alpha))}=\frac{2 \pi D}{Z^{2} \alpha}\left(\frac{\sinh (\pi \alpha)}{1+\cosh (\pi \alpha)}\right)=\frac{2 \pi D}{Z^{2} \alpha}\left(\frac{\cosh (\pi \alpha)-1}{\sinh (\pi \alpha)}\right)
\end{aligned}
$$

which is real and positive for real $\alpha$. 
In the general case $c_{1} \neq 0$, under the same changes of variables, integrals (4.5) yield

$$
\begin{aligned}
\Delta x_{C} \mp \mathrm{i} \Delta y_{C}= & 2 \frac{\sigma \sqrt{D}}{Z} \int_{0}^{\pi / 2} \cos \left(2 c_{1}(u-\pi / 4)\right)(\cos u)^{ \pm \mathrm{i} \alpha}(\sin u)^{ \pm \mathrm{i} \alpha} \mathrm{d} u \\
= & 2 \frac{\sigma \sqrt{D}}{Z}\left(2^{\mp \mathrm{i} \alpha}\right) \int_{0}^{\pi / 2} \cos \left(2 c_{1}(u-\pi / 4)\right)(\sin (2 u))^{ \pm \mathrm{i} \alpha} \mathrm{d} u=\{w=2 u\} \\
= & \frac{\sigma \sqrt{D}}{Z}\left(2^{\mp \mathrm{i} \alpha}\right) \int_{0}^{\pi} \cos \left(c_{1}(w-\pi / 2)\right)(\sin w)^{ \pm \mathrm{i} \alpha} \mathrm{d} w \\
= & \frac{\sigma \sqrt{D}}{Z}\left(2^{\mp \mathrm{i} \alpha}\right)\left[\cos \left(\frac{c_{1} \pi}{2}\right) \int_{0}^{\pi} \cos \left(c_{1} w\right)(\sin w)^{ \pm \mathrm{i} \alpha} \mathrm{d} w\right. \\
& \left.+\sin \left(\frac{c_{1} \pi}{2}\right) \int_{0}^{\pi} \sin \left(c_{1} w\right)(\sin w)^{ \pm \mathrm{i} \alpha} \mathrm{d} w\right]
\end{aligned}
$$

The last two integrals on the right-hand side can be calculated in terms of the Beta function by applying the general formulae $[9]^{6}$

$$
\begin{aligned}
& \int_{0}^{\pi} \cos \left(c_{1} w\right)(\sin w)^{\nu-1} \mathrm{~d} w=\frac{\pi \cos \left(\frac{c_{1} \pi}{2}\right)}{2^{\nu-1} v B\left(\frac{v+c_{1}+1}{2}, \frac{v-c_{1}+1}{2}\right)}, \\
& \int_{0}^{\pi} \sin \left(c_{1} w\right)(\sin w)^{\nu-1} \mathrm{~d} w=\frac{\pi \sin \left(\frac{c_{1} \pi}{2}\right)}{2^{\nu-1} \nu B\left(\frac{v+c_{1}+1}{2}, \frac{v-c_{1}+1}{2}\right)},
\end{aligned}
$$

$v$ being a complex number with a positive real part. Then, after setting $v=1 \pm \mathrm{i} \alpha,($ A.5) gives

$$
\Delta x_{C} \mp \mathrm{i} \Delta y_{C}=\frac{\sigma \sqrt{D}}{Z} \frac{4^{\mp \mathrm{i} \alpha} \pi}{(1 \pm \mathrm{i} \alpha) B\left(1+\frac{c_{1} \pm \mathrm{i} \alpha}{2}, 1+\frac{-c_{1} \pm \mathrm{i} \alpha}{2}\right)} .
$$

Therefore, using (A.3) and (A.4), we find that the square of the distance is given by

$$
\begin{aligned}
d^{2} & =\left(\Delta x_{C}\right)^{2}+\left(\Delta y_{C}\right)^{2}=\frac{D \pi^{2}}{Z^{2}\left(1+\alpha^{2}\right)}\left[\frac{1}{B\left(1+\frac{c_{1}+\mathrm{i} \alpha}{2}, 1+\frac{-c_{1}+\mathrm{i} \alpha}{2}\right) B\left(1+\frac{c_{1}-\mathrm{i} \alpha}{2}, 1+\frac{-c_{1}-\mathrm{i} \alpha}{2}\right)}\right] \\
& =\frac{D \pi^{2}}{Z^{2}\left(1+\alpha^{2}\right)}\left[\frac{\Gamma(2+\mathrm{i} \alpha) \Gamma(2-\mathrm{i} \alpha)}{\Gamma\left(1+\frac{c_{1}+\mathrm{i} \alpha}{2}\right) \Gamma\left(1+\frac{-c_{1}+\mathrm{i} \alpha}{2}\right) \Gamma\left(1+\frac{c_{1}-\mathrm{i} \alpha}{2}\right) \Gamma\left(1+\frac{-c_{1}-\mathrm{i} \alpha}{2}\right)}\right] \\
& =\frac{D \pi^{2}}{Z^{2}\left(1+\alpha^{2}\right)}\left[\frac{\alpha^{2}\left(1+\alpha^{2}\right) \Gamma(\mathrm{i} \alpha) \Gamma(-\mathrm{i} \alpha)}{\left(\frac{c_{1}^{2}+\alpha^{2}}{4}\right)^{2}\left(\Gamma\left(\frac{c_{1}+\mathrm{i} \alpha}{2}\right) \Gamma\left(-\frac{c_{1}+\mathrm{i} \alpha}{2}\right)\right)\left(\Gamma\left(\frac{c_{1}-\mathrm{i} \alpha}{2}\right) \Gamma\left(-\frac{c_{1}-\mathrm{i} \alpha}{2}\right)\right)}\right] \\
& =\frac{D \pi^{2} \alpha^{2}}{Z^{2}}\left[\frac{\sin \left(\pi\left(\frac{c_{1}+\mathrm{i} \alpha}{2}\right)\right) \sin \left(\pi\left(\frac{c_{1}-\mathrm{i} \alpha}{2}\right)\right)}{-\mathrm{i} \alpha \pi\left(\frac{c_{1}^{2}+\alpha^{2}}{4}\right) \sin (\mathrm{i} \alpha \pi)}\right] \\
& =\frac{4 \pi D}{Z^{2}}\left(\frac{\alpha}{c_{1}^{2}+\alpha^{2}}\right)\left(\frac{\sin ^{2}\left(\frac{c_{1} \pi}{2}\right) \cosh \left(\frac{\alpha \pi}{2}\right)+\cos ^{2}\left(\frac{c_{1} \pi}{2}\right) \sinh ^{2}\left(\frac{\alpha \pi}{2}\right)}{\sinh (\alpha \pi)}\right),
\end{aligned}
$$

and the last expression simplifies to (4.6).

6 The original references for these formulae are [18] and [27]. 


\section{References}

[1] Arnold V I, Kozlov V V and Neishtadt A I 1985 Mathematical aspects of classical and celestial mechanics Itogi Nauki i Tekhniki (Sovr. Probl. Mat. Fundamental'nye Napravleniya vol 3) (Moscow: VINITI)

Arnold V I, Kozlov V V and Neishtadt A I 1989 Encyclopadia of Mathematical Sciences vol 3 (Berlin: Springer) (Engl. Transl.)

[2] Borisov A V, Mamaev I S and Ramodanov S M 2003 Motion of a circular cylinder and $n$ point vortices in a perfect fluid Regular Chaotic Dyn. 8 449-62

[3] Borisov A V and Mamaev I S 2009 The dynamics of a Chaplygin sleigh Prikl. Mat. Mekh. 73 219-25 (in Russian)

Borisov A V and Mamaev I S 2009 J. Appl. Math. Mech. 73 156-61 (Engl. Transl.)

[4] Bloch A M 2003 Nonholonomic Mechanics and Control (New York: Springer)

[5] Der Schlitten C C 1933 Z. Angew. Math. Mech. 13 71-6

[6] Chaplygin S A 1911 On the theory of motion of nonholonomic systems. The theorem of the reducing multiplier Math. Sb. 28 303-14 (in Russian)

[7] Erdélyi A, Magnus W, Oberhettinger F and Tricomi F G 1981 Higher Transcendental Functions vol 1 (Melbourne, FL: Krieger)

[8] García-Naranjo L C 2007 Reduction of almost Poisson brackets for nonholonomic systems on Lie groups Regular Chaotic Dyn. 14 365-88

[9] Gradshteyn I S and Ryzhik I M 2007 Table of integrals, series, and products 7th edn, ed A Jeffrey and D Zwillinger (Amsterdam: Elsevier)

[10] Jovanović B 1988 Nonholonomic geodesic flows on Lie groups and the integrable Suslov problem on $S O(4)$ J. Phys. A: Math. Gen. 31 1415-22

[11] Jovanović B 2003 Some multidimensional integrable cases of nonholonomic rigid body dynamics Regular Chaotic Dyn. 8 125-32

[12] Kanso E, Marsden J E, Rowley C W and Melli-Huber J B 2005 Locomotion of articulated bodies in a perfect fluid J. Nonlinear Sci. 15 255-89

[13] Kozlov V V 1982 The dynamics of systems with nonintegrable constraints: I, II. Vestnik Moskov. Univ. Ser. I Mat. Mekh. 112 92-100 and 86 70-76 (in Russian)

[14] Kozlov V V 1985 On the integrability theory of equations of nonholonomic mechanics Adv. Mech. 8 85-107 (in Russian)

Kozlov V V 2002 Reg. Chaot. Dyn. 7 161-76 (Engl. Transl.)

[15] Kozlov V V 1988 Invariant measures of the Euler-Poincaré equations on Lie algebras Funkt. Anal. Prilozh. 22 69-70 (in Russian)

Kozlov V V 1988 Funct. Anal. Appl. 22 58-9 (Engl. Transl.)

[16] Lamb H 1932 Hydrodynamics 6th edn (New York: Dover)

[17] Landau L D and Lifshitz E M 1976 Mechanics 3rd edn (Oxford: Butterworth-Heinemann)

[18] Lobachevskiy N I 1946-1951 Polnoye Sobraniye Sochineniy (Complete works) vols 1, 2 and 5 (Moscow: Gostekhizdat) (in Russian)

[19] Neimark Ju I and Fufaev N A 1972 Dynamics of Nonholonomic Systems (Translations of Mathematical Monographs vol 33) (Providence, RI: American Mathematical Society)

[20] Novikov S P 1981 Variational methods and periodic solutions of equations of Kirchhoff type: II Funktsional. Anal. i Prilozhen. 15 37-52, 96 (in Russian)

[21] Osborne J and Zenkov D 2005 Steering the Chaplygin sleigh using a moving mass Proc. Conf. on Decision and Control (CDC-ECC)

[22] Ramodanov S M 2000 Motion of a circular cylinder and a vortex in an ideal fluid Regular Chaotic Dyn. $633-8$

[23] Rand R H and Ramani D V 2000 Relaxing nonholonomic constraints Proc. of the First Int. Symp. on Impact and Friction of Solids, Structures, and Intelligent Machines ed A Guran (Singapore: World Scientific) pp 113-6

[24] Suslov G 1951 Theoretical Mechanics (Moscow: Gostekhizdat) (in Russian)

[25] Tatarinov Ya V 1988 Separation of variables and new topological phenomena in holonomic and nonholonomic systems Tr. Sem. Vektor. Tenzor. Anal. 160-74 (in Russian)

[26] Vankerschaver J, Kanso E and Marsden J E 2009 The geometry and dynamics of interacting rigid bodies and point vortices J. Geom. Mech. $1223-66$

[27] Watson G N 1966 A Treatise on the Theory of Bessel Functions 2nd edn (London: Cambridge University Press)

[28] Zenkov D V and Bloch A M 2000 Dynamics of the $n$-dimensional Suslov problem J. Geom. Phys. 34 121-36 